Annals of Pure and Applied Mathematics

Vol. 20, No. 1, 2019, 49-54

ISSN: 2279-087X (P), 2279-0888(online)

Published on 16 September 2019

www.researchmathsci.org

DOI: http://dx.doi.org/10.22457/apam.631v20n1a7

Annals of

Pure and Applied

Mathematics

\title{
Connectivity Neighborhood Dakshayani Indices of POPAM Dendrimers
}

\author{
V.R.Kulli \\ Department of Mathematics \\ Gulbarga University, Gulbarga 585 106, India \\ E-mail:vrkulli@gmail.com
}

Received 20 August 2019; accepted 15 September 2019

\begin{abstract}
In Chemical Graph Theory, connectivity indices are applied to measure the chemical characteristics of chemical compounds. In this study, we introduce the sum connectivity neighborhood Dakshayani index, product connectivity neighborhood Dakshayani index, atom bond connectivity neighborhood Dakshayani index, geometric arithmetic neighborhood Dakshayani index and arithmetic-geometric neighborhood Dakshayani index of a molecular graph. We compute these connectivity neighborhood Dakshayani indices of POPAM dendrimers.
\end{abstract}

Keywords: neighborhood Dakshayani indices, connectivity neighborhood Dakshayani indices, dendrimer.

AMS Mathematics Subject Classification (2019): 05C07, 05C35, $05 C 76$

\section{Introduction}

Throughout this paper, we consider only finite, connected, undirected graphs without loops and multiple edges. The degree of a vertex, denoted by $d_{G}(v)$, is the number of vertices adjacent to $v$. The set of all vertices which adjacent to $v$ is called open neighborhood of $v$ and denoted by $N_{G}(v)$. The closed neighborhood of $v$ is the set $N_{G}[v]=$ $N_{G}(v) \cup\{v\}$. The set $N_{G}[v]$ is the set of closed neighborhood vertices of $v$. Let $D_{G}(v)=d_{G}(v)+\sum_{u \in N_{G}(v)} D_{G}(u)$ be the degree sum of closed neighborhood vertices of $v$.

We refer [1] for undefined terminologies and notations from graph theory.

A molecular graph or a chemical graph is a graph such that the vertices correspond to the atoms and edges to the bonds. Chemical Graph Theory is a breach of Mathematical Chemistry whose focus of interest is to finding topological indices or graph indices of a molecular graph which correlate well with chemical properties of the chemical molecules. Numerous graph indices have been considered in Chemistry and have found some applications in QSPR/QSAR research see [2,3]

In [4], Kulli introduced the first and second neighborhood Dakshayani indices of a graph, defined as

$$
N D_{1}(G)=\sum_{u v \in E(G)}\left[D_{G}(u)+D_{G}(v)\right], \quad N D_{2}(G)=\sum_{u v \in E(G)} D_{G}(u) D_{G}(v) .
$$


The first and second hyper neighborhood Dakshayani indices were proposed and studied by Kulli in [4], defined as

$$
H N D_{1}(G)=\sum_{u v \in E(G)}\left[D_{G}(u)+D_{G}(v)\right]^{2}, \quad H N D_{2}(G)=\sum_{u v \in E(v)}\left[D_{G}(u) D_{G}(v)\right]^{2} \text {. }
$$

Motivated by the definitions of the connectivity indices, we now introduce the connectivity neighborhood indices as follows.

The sum and product connectivity neighborhood Dakshayani indices of a graph $G$ are defined as

$$
\begin{aligned}
& \operatorname{SND}(G)=\sum_{u v \in E(G)} \frac{1}{\sqrt{D_{G}(u)+D_{G}(v)}} . \\
& \operatorname{PND}(G)=\sum_{u v \in E(G)} \frac{1}{\sqrt{D_{G}(u) D_{G}(v)}} .
\end{aligned}
$$

The atom bond connectivity $(\mathrm{ABC})$ neighborhood Dakshayani index of a graph $G$ is defined as

$$
A B C N D(G)=\sum_{u v \in E(G)} \sqrt{\frac{D_{G}(u)+D_{G}(v)-2}{D_{G}(u) D_{G}(v)}} .
$$
defined as

The geometric-arithmetic neighborhood Dakshayani index of a graph $G$ is

$$
G A N D(G)=\sum_{u v \in E(G)} \frac{2 \sqrt{D_{G}(u) D_{G}(v)}}{D_{G}(u)+D_{G}(v)} .
$$
defined as

The arithmetic-geometric neighborhood Dakshayani index of a graph $G$ is

$$
A G N D(G)=\sum_{u v \in E(G)} \frac{D_{G}(u)+D_{G}(v)}{2 \sqrt{D_{G}(u) D_{G}(v)}} .
$$

The reciprocal neighborhood Dakshayani index of a graph $G$ is defined as

$$
R N D(G)=\sum_{u v \in E(G)} \sqrt{D_{G}(u) D_{G}(v)}
$$
defined as

The general first and second neighborhood Dakshayani indices of a graph $G$ are

$$
\begin{gathered}
N D_{1}^{a}(G)=\sum_{u v \in E(G)}\left[D_{G}(u)+D_{G}(v)\right]^{a}, \\
N D_{2}^{a}(G)=\sum_{u v \in E(G)}\left[D_{G}(u) D_{G}(v)\right]^{a},
\end{gathered}
$$

where $a$ is a real number.

Recently, some now connectivity indices were introduced and studied such as sum connectivity index [5], sum connectivity Revan index [6], sum connectivity Gourava index [7], atom bond connectivity Kulli-Basava index [8], sum and product connectivity F-indices [9], multiplicative connectivity $K V$ indices [10], sum connectivity leap index [11], connectivity $K V$ indices [12]. Very recently some new variants of neighborhood Dakshayani indices were proposed and studied such as $F$-neighborhood Dakshayani 
Connectivity Neighborhood Dakshayani Indices of POPAM Dendrimers

index [13], square neighborhood Dakshayani index [14], multiplicative neighborhood indices [15]. determined.

In this paper, some neighborhood Dakshayani indices of $P O P A M$ dendrimers are

\section{Results for POPAM Dendrimers}

In this section, we focus on the graph of POPAM dendrimer. This family of dendrimers is symbolized by $\mathrm{POD}_{2}[n]$, where $\mathrm{n}$ is the steps of growth in POPAM dendrimers. A graph of $\mathrm{POD}_{2}[2]$ is shown in Figure 1.

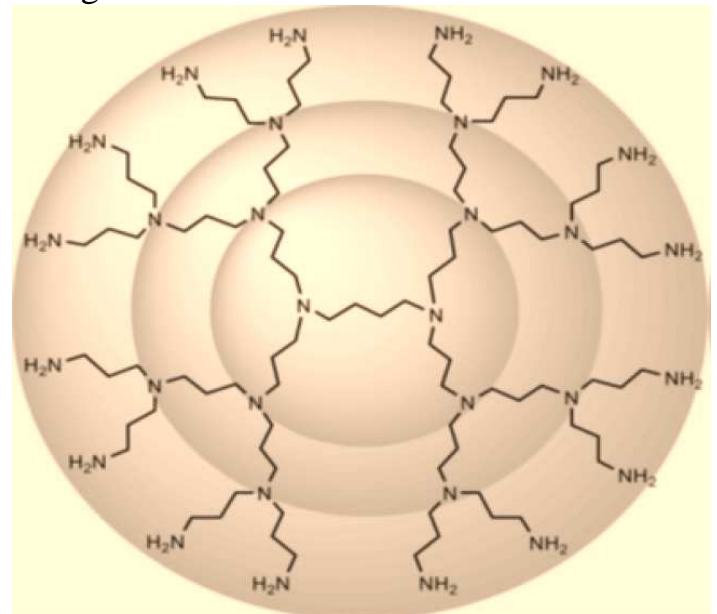

Figure 1: Graph of $\mathrm{POD}_{2}[2]$

Let $G$ be a graph of a $\mathrm{POD}_{2}[n]$ dendrimer. By calculation $G$ has $2^{n+5}-10$ vertices and $2^{n+5}$ - 11 edges. The edge partition of $G$ based on the degree gum of closed neighborhood end vertices of each edge is obtained as given in Table 1.

Table 1: Edge partition of $\mathrm{POD}_{2}[n]$

\begin{tabular}{lccccc}
\hline$D_{G}(u), D_{G}(v) \backslash u v \in E(G)$ & $(3,5)$ & $(5,6)$ & $(6,6)$ & $(6,7)$ & $(7,9)$ \\
\hline Number of edges & $2^{n+2}$ & $2^{n+2}$ & 1 & $3 \times 2^{n+2}-6$ & $3 \times 2^{n+2}-6$ \\
\hline
\end{tabular}

Theorem 1. The general first neighborhood Dakshayani index of a POPAM dendrimer $\mathrm{POD}_{2}[n]$ is

$$
N D_{2}^{a}\left[P O D_{2}(n)\right]=\left(8^{a}+11^{a}+3 \times 13^{a}+3 \times 16^{a}\right) 2^{n+2}+\left(12^{a}-6 \times 13^{a}-6 \times 16^{a}\right)
$$

Proof: Let $G$ be a graph of $P O P A M$ dendrimer $\mathrm{POD}_{2}[n]$. By using equation (4) and Table 1 , we obtain

$$
\begin{aligned}
& N D_{1}^{a}\left(P O D_{2}[n]\right)=\sum_{u v \in E(G)}\left[D_{G}(u)+D_{G}(v)\right]^{a} \\
& =(3+5)^{a} 2^{n+2}+(5+6)^{a} 2^{n+2}+(6+6)^{a}+(6+7)^{a}\left(3 \times 2^{n+2}-6\right)+(7+9)^{a}\left(3 \times 2^{n+2}-6\right) \\
& =\left(8^{a}+11^{a}+3 \times 13^{a}+3 \times 16^{a}\right) 2^{n+2}+\left(12^{a}-6 \times 13^{a}-6 \times 16^{a}\right) .
\end{aligned}
$$


V.R.Kulli

We obtain the following results by using Theorem 1 .

Corollary 1.1. If $\mathrm{POD}_{2}[n]$ is graph of $P O P A M$ dendrimer, then

i) $\quad N D_{1}\left(P_{2}[n]\right)=106 \times 2^{n+2}-162$.

ii) $\quad H N D_{1}\left(P_{2}[n]\right)=1460 \times 2^{n+2}-2406$.

iii) $\quad \operatorname{SND}\left(\mathrm{POD}_{2}[n]\right)=\left(\frac{1}{\sqrt{8}}+\frac{1}{\sqrt{11}}+\frac{3}{\sqrt{13}}+\frac{3}{4}\right) 2^{n+2}+\left(\frac{1}{\sqrt{12}}-\frac{6}{\sqrt{13}}-\frac{6}{4}\right)$

Proof: Put $a=1,2,-1 / 2$ in equation (6), we get the required results.

Theorem 2. The general second neighborhood Dakshayani index of POPAM dendrimer $\mathrm{POD}_{2}[n]$ is

$N D_{2}^{a}\left[P O D_{2}(n)\right]=\left(15^{a}+30^{a}+3 \times 42^{a}+3 \times 63^{a}\right) 2^{n+2}+\left(36^{a}-6 \times 42^{a}-6 \times 63^{a}\right)(7)$

Proof: Let $G$ be a graph of $P O P A M$ dendrimer $P O D_{2}[n]$. By using equation (5) and Table 1 , we deduce

$$
\begin{aligned}
& N D_{1}^{a}\left(P O D_{2}[n]\right)=\sum_{u v \in E(G)}\left[D_{G}(u) D_{G}(v)\right]^{a} \\
& =(3 \times 5)^{a} 2^{n+2}+(5 \times 6)^{a} 2^{n+2}+(6 \times 6)^{a}+(6 \times 7)^{a}\left(3 \times 2^{n+2}-6\right)+(7 \times 9)^{a}\left(3 \times 2^{n+2}-6\right) \\
& =\left(15^{a}+30^{a}+3 \times 42^{a}+3 \times 63^{a}\right) 2^{n+2}+\left(36^{a}-6 \times 42^{a}-6 \times 63^{a}\right) .
\end{aligned}
$$

Corollary 2.1. Let $\mathrm{POD}_{2}[n]$ be a graph of $P O P A M$ dendrimer. Then

i) $\quad N D_{2}\left(P O D_{2}[n]\right)=360 \times 2^{n+2}-594$.

ii) $\quad H N D_{2}\left(\mathrm{POD}_{2}[n]\right)=18324 \times 2^{n+2}-33102$.

iii) $\operatorname{PND}\left(\operatorname{POD}_{2}[n]\right)=\left(\frac{1}{\sqrt{15}}+\frac{1}{\sqrt{30}}+\frac{3}{\sqrt{42}}+\frac{3}{\sqrt{63}}\right) 2^{n+2}+\left(\frac{1}{6}-\frac{6}{\sqrt{42}}-\frac{6}{\sqrt{63}}\right)$.

iv) $R N D\left(P O D_{2}[n]\right)=(\sqrt{15}+\sqrt{30}+3 \times \sqrt{42}+3 \times \sqrt{63}) 2^{n+2}+(6-6 \times \sqrt{42}-3 \times \sqrt{63})$.

Proof: Put $a=1,2,-1 / 2,1 / 2$ in equation (7), we obtain the desired results.

Theorem 3. The atom bond connectivity neighborhood Dakshayani index of $P O D_{2}[n]$ is

$$
A B C N D\left(P O D_{2}[n]\right)=\left(\sqrt{\frac{2}{5}}+\sqrt{\frac{3}{10}}+3 \sqrt{\frac{11}{42}}+\sqrt{2}\right) 2^{n+2}+\left(\sqrt{\frac{5}{18}}-6 \sqrt{\frac{11}{42}}-2 \sqrt{2}\right)
$$

Proof: Let $G=\mathrm{POD}_{2}[n]$. By using equation (1) and Table 1, we have

$$
\begin{aligned}
& A B C N D\left(P_{2}[n]\right)=\sum_{u v \in E(G)} \sqrt{\frac{D_{G}(u)+D_{G}(v)-2}{D_{G}(u) D_{G}(v)}} \\
& =\left(\sqrt{\frac{3+5-2}{3 \times 5}}\right) 2^{n+2}+\left(\sqrt{\frac{5+6-2}{5 \times 6}}\right) 2^{n+2}+\left(\sqrt{\frac{6+6-2}{6 \times 6}}\right) \\
& +\left(\sqrt{\frac{6+7-2}{6 \times 7}}\right)\left(3 \times 2^{n+2}-6\right)+\left(\sqrt{\frac{7+9-2}{7 \times 9}}\right)\left(3 \times 2^{n+2}-6\right)
\end{aligned}
$$


Connectivity Neighborhood Dakshayani Indices of POPAM Dendrimers

$$
=\left(\sqrt{\frac{2}{5}}+\sqrt{\frac{3}{10}}+3 \sqrt{\frac{11}{42}}+\sqrt{2}\right) 2^{n+2}+\left(\sqrt{\frac{5}{18}}-6 \sqrt{\frac{11}{42}}-2 \sqrt{2}\right) .
$$

Theorem 4. The geometric-arithmetic neighborhood Dakshayani index of $\mathrm{POD}_{2}[n]$ is $\operatorname{GAND}\left(\right.$ POD $\left._{2}[n]\right)=\left(\frac{\sqrt{15}}{4}+\frac{2 \sqrt{30}}{11}+\frac{6 \sqrt{42}}{13}+\frac{9 \sqrt{7}}{8}\right) 2^{n+2}+\left(1-\frac{12 \sqrt{42}}{13}-\frac{18 \sqrt{7}}{8}\right)$

Proof: Let $G=\mathrm{POD}_{2}[n]$. From equation (2) and by using Table 1, we obtain

$$
\begin{aligned}
& \text { GAND }\left(\text { POD }_{2}[n]\right)=\sum_{u v \in E(G)} \frac{2 \sqrt{D_{G}(u) D_{G}(v)}}{D_{G}(u)+D_{G}(v)} \\
& =\left(\frac{2 \sqrt{3 \times 5}}{3+5}\right) 2^{n+2}+\left(\frac{2 \sqrt{5 \times 6}}{5+6}\right) 2^{n+2}+\left(\frac{2 \sqrt{6 \times 6}}{6+6}\right) \\
& +\left(\frac{2 \sqrt{6 \times 7}}{6+7}\right)\left(3 \times 2^{n+2}-6\right)+\left(\frac{2 \sqrt{7 \times 9}}{7+9}\right)\left(3 \times 2^{n+2}-6\right) \\
& =\left(\frac{\sqrt{15}}{4}+\frac{2 \sqrt{30}}{11}+\frac{6 \sqrt{42}}{13}+\frac{9 \sqrt{7}}{8}\right) 2^{n+2}+\left(1-\frac{12 \sqrt{42}}{13}-\frac{18 \sqrt{7}}{8}\right) .
\end{aligned}
$$

Theorem 5. The arithmetic-geometric neighborhood Dakshayani index of $\mathrm{POD}_{2}[n]$ is $A G N D\left(P_{2}[n]\right)=\left(\frac{4}{\sqrt{15}}+\frac{11}{2 \sqrt{30}}+\frac{39}{2 \sqrt{42}}+\frac{8}{\sqrt{7}}\right) 2^{n+2}+\left(1-\frac{39}{\sqrt{42}}-\frac{16}{\sqrt{7}}\right)$

Proof: Let $G=P O D_{2}[n]$. By using equation (3) and Table 1, we deduce

$$
\begin{aligned}
& A G N D\left(P O D_{2}[n]\right)=\sum_{u v \in E(G)} \frac{D_{G}(u)+D_{G}(v)}{2 \sqrt{D_{G}(u) D_{G}(v)}} \\
& =\left(\frac{3+5}{2 \sqrt{3 \times 5}}\right) 2^{n+2}+\left(\frac{5+6}{2 \sqrt{5 \times 6}}\right) 2^{n+2}+\left(\frac{6+6}{2 \sqrt{6 \times 6}}\right) \\
& +\left(\frac{6+7}{2 \sqrt{6 \times 7}}\right)\left(3 \times 2^{n+2}-6\right)+\left(\frac{7+9}{2 \sqrt{7 \times 9}}\right)\left(3 \times 2^{n+2}-6\right) \\
& =\left(\frac{4}{\sqrt{15}}+\frac{11}{2 \sqrt{30}}+\frac{39}{2 \sqrt{42}}+\frac{8}{\sqrt{7}}\right) 2^{n+2}+\left(1-\frac{39}{\sqrt{42}}-\frac{16}{\sqrt{7}}\right) .
\end{aligned}
$$

Acknowledgement. The author is thankful to the referee for useful suggestions.

\section{REFERENCES}

1. V.R.Kulli, College Graph Theory, Vishwa International Publications, Gulbarga, India (2012).

2. V.R. Kulli, Multiplicative Connectivity Indices of Nanostructures, LAP LEMBERT Academic Publishing, (2018).

3. I.Gutman and O.E.Polansky, Mathematical Concepts in Organic Chemistry, Springer, Berlin (1986).

4. V.R.Kulli, Neighborhood Dakshayani indices, International Journal of Mathematical Archive, 10(7) (2019) 23-31. 


\section{V.R.Kulli}

5. B.Zhou and N.Trinajstic, On a novel connectivity index, J. Math. Chem., 46 (2009) 1252-1270.

6. V.R.Kulli, The sum connectivity Revan index of silicate and hexagonal networks, Annals of Pure and Applied Mathematics, 14(3) (2017) 401-406.

7. V.R.Kulli, On the sum connectivity Gourava index, International Journal of Mathematical Archive, 8(7) (2017) 211-217.

8. V.R.Kulli, New connectivity topological indices, Annals of Pure and Applied Mathematics, 20(1) (2019) 1-8.

9. V.R.Kulli, F-indices of chemical networks, International Journal of Mathematics Archive, 10(3) (2019) 21-30.

10. V.R.Kulli, Multiplicative connectivity KV indices of dendrimers, Journal of Mathematics and Informatics, 15 (2019) 1-7.

11. V.R.Kulli, Sum connectivity leap index and geometric-arithmetic, leap index of certain windmill graphs, Journal of Global Research in Mathematical Archives, 6(1) (2019) 15-20.

12. V.R.Kulli, On connectivity $\mathrm{KV}$ indices of certain families of dendrimers, International Journal of Mathematical Archive, 10(2) (2019) 14-17.

13. V.R.Kulli, Neighborhood Dakshayani indices of nanostructures, International Journal of Current Research in Science and Technology, 5(7) (2019) 1-12.

14. V.R.Kulli, F1-neighborhood and square neighborhood Dakshayani indices some nanostructures, International Journal of Engineering Sciences and Research Technology, 8(8) (2019) 126-138.

15. V.R.Kulli, Multiplicative neighborhood indices, Annals of Pure and Applied Mathematics, 19(2) (2019) 175-181. 